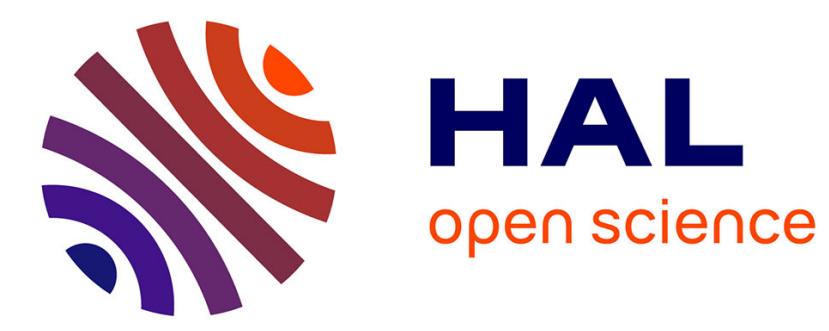

\title{
Effects of grain boundaries on the mechanical behaviour of grains in polycrystals \\ C. Rey
}

\section{To cite this version:}

C. Rey. Effects of grain boundaries on the mechanical behaviour of grains in polycrystals. Revue de Physique Appliquée, 1988, 23 (4), pp.491-500. 10.1051/rphysap:01988002304049100 . jpa-00245796

\section{HAL Id: jpa-00245796 https://hal.science/jpa-00245796}

Submitted on 1 Jan 1988

HAL is a multi-disciplinary open access archive for the deposit and dissemination of scientific research documents, whether they are published or not. The documents may come from teaching and research institutions in France or abroad, or from public or private research centers.
L'archive ouverte pluridisciplinaire HAL, est destinée au dépôt et à la diffusion de documents scientifiques de niveau recherche, publiés ou non, émanant des établissements d'enseignement et de recherche français ou étrangers, des laboratoires publics ou privés. 


\title{
Effects of grain boundaries on the mechanical behaviour of grains in polycrystals
}

\author{
C. Rey
}

Laboratoire PMTM CNRS, Université Paris-Nord, Avenue J.B. Clément, 93430 Villetaneuse, France

(Reçu le 15 juin 1987, révisé le 18 septembre 1987, accepté le 15 octobre 1987)

Résumé. - Les effets des joints de grain sur la déformation plastique des métaux purs polycrysta1lins déformés à froid sont étudiés à l'échelle du grain et de l'agregat.

Dans la première partie, on décrit les moyens permettant de passer du comportement local au comportement global. L'accent est mis sur la nécessité de prendre en compte les effets d'interactions entre grains.

Les résultats expérimentaux concernant le comportement global sont résumés dans le paragraphe 2. On s'est limité à l'effet de la taille de grain sur les courbes de durcissement et à la formation des hétérogénéités intragranulaires de déformation plastisque.

Le paragraphe 3 est basé sur l'observation expérimentale des effets des joints et des jonctions de grains sur la réponse du grain. La formation et l'évolution des hétérogénéités de déformation plastique sont analysées à l'échelle du grain.

Abstract. - The effects of grain boundaries on the plasticity of metallic polycrystals loaded at room temperature are studied at two levels, the level of the grain and the level of the aggregate.

In the first section, the methods allowing the transition from the local level to the global one are summarized. We lay stress on the necessity to take into account interactions effects between grains.

Experimental results about the global behaviour are reported in the section 2 . We have restricted the results to the effects of the grain size on the flowstress curve, and to the description of some inhomogeneous slip patterns.

The section 3 is based on the experimentai observations of grain boundaries and junctions of grains effects on the plastic response of grains of a polycrystal. The formation and evolution of the plastic inhomogeneities are analyzed at the grain level.

\section{Introduction.}

This paper deals with the mechanical behaviour of metallic polycrystals. The complexity of the macroscopic behaviour lies in the fact that the internal state of the material is continuously charying during the plastic flow. This change corresponds to a simultaneous twofold evolution combining the effects of the microstructure evolution (such as dislocations distribution inside the grains, formation of cell structure) and some more macroscopic phenomena leading to the formation of inhomogeneous slip patterns and even to the lattice orientation evolution.
Many approaches were undertaken to connect the macroscopic behaviour to the material structural features and especially those bound to grain boundaries. The main difficulties were to determine the most significant physical parameters or mechanisms responsible for the macroscopic behaviour. Such analyses were carried out at different physical levels : microscopic (dislocation level), medium (slip band level to grain level) and macroscopic (aggregate leve1).

The aim of the present section is to collect, in a short summary, some previous results related to these different levels. 
Section 2 is devoted to a review of experimental works and to modellings concerning the different parameters ruling the macroscopic behaviour of aggregates.

In section 3 we focus on the mechanisms of the plastic deformation induced by grains boundaries.

1.1. Microscopic and medium levels.

Let us start our review from the microscopic scale and gradually increase the investigation level to reach the macroscopical scale. As a first step, we consider the interactions between the defects of the grain boundary and the lattice dislocations. At low temperature, such interactions concern a very small part of the grain and therefore cannot play an important part in the whole grain macroscopic behaviour. Thus they will be not hereafter considered.

Grain boundaries are usually assumed to be at least a source of dislocations, an obstacle to the plastic glide and a source of internal stresses bound to the incompatibility of the plastic deformation between two neighbouring grains.

- A source of dislocations : under applied stresses, grain boundary singularities,such as ledges, originate large local stresses able to activate lattice dislocations. Such phenomena contribute to harden the vicinity of grain boundaries.

- Grain boundaries act as an obstacle to the plastic glide and ensure, at low temperature, the cohesion of different plastified grains. In both cases, conditions on the compatibility of the plastic strain at the grain boundaries have to be fulfilled. In the microplasticity stage, usually only few slip bands are activated in a grain and the corresponding dislocations pile up on the grain boundary. The local compatibility conditions are fulfilled either by addition of an extra elastic strain field or (and) by activation of some extra slip systems localized in the adjacent grain at the head of each slip band /1/.

With increasing deformation (plasticity stage) the slip pattern becomes more homogeneous and the compatibility conditions can be written assuming that grains are uniformly plastified along the relevant grain boundary.

It will be shown in section 3 that the mechanisms of accommodation of the plastic incompatibilities are complex and lead to a weaker hardening than generally admitted.

\subsection{Macroscopic level.}

The local effects of grain boundaries on the plastic deformation of grains must be expressed in terms of local stress, strain and rotation fields $(\bar{\sigma}, \bar{\varepsilon}$ and $\bar{w}$ respectively). These effects must be a function of the grain deformation mechanisms and of the typical features of grain boundaries (lattice misorientation, position of grain boundaries).

The transition from this basic level to the macroscopic one, involves two kinds of difficulties :

first, the determination of statistically significant effects : in an aggregate we cannot take into account an individual description of grain boundaries. It is necessary to simplify the data by considering only important statistical effects.

Second, the determination of the interactions between grains of various shapes, grain sizes and orientations :the local stress field calculated in simple geometrical cases such as bicrystal, tricrystal, multicrystal, is different from the actual one in any grain of an aggregate. The description of the aggregate behaviour needs a careful investigation and an estimation of the interactions between adjoining grains.

Let us examine now the means of spanning the gap between the aforementioned local and global levels :according to the self consistent scheme of Kröner based on Eshelby's inclusion problem /2/ /3/ $14 /$, one considers the interaction fo a given grain with a plastic strain $\bar{\varepsilon}^{\mathrm{P}}$ and the polycristalline matrix.

The detailed distribution of the plastic strain in the matrix is replaced by an averaged one, fixed by the macroscopic value $\overline{\mathrm{E}}^{\mathrm{P}}$. The matrix is considered as an homogeneous medium with the same mechanical behaviour as the polycrystal one. Assuming a spherically shaped grain and an uniformly plastified infinite matrix stressed by $\bar{\Sigma}$ at infinite distance, one obtains the following interaction law between the local fields ( $\bar{\sigma}$ and $\bar{\varepsilon}^{\mathrm{P}}$ ) inside the inclusion and the global fields $\left(\bar{\Sigma}, \overline{\mathrm{E}}^{\mathrm{P}}\right)$ for the case of isotropic elasticity:

$$
\begin{aligned}
& \bar{\sigma}=\bar{\Sigma}+2 \mu(1-\beta)\left(\overline{\mathrm{E}}^{\mathrm{P}}-\bar{\varepsilon}^{\mathrm{P}}\right) \\
& \beta=\frac{2(4-5 \nu)}{15(1-\nu)} \\
& \mu \text { is the shear modulus } \\
& \nu \text { is the Poisson's ratio. }
\end{aligned}
$$

This interaction law assumes an elastic accommodation of the plastic incompatibility 
between the inclusion and the matrix.Note that such an approximation leads to an overestimation of internal stresses $\bar{\sigma}$.

A similar treatment was performed taking into account the plastic flow of the matrix $/ 5 / / 6 /$. In this case the hereabove interaction law can be written under a derivative form, in the following way :

$$
\stackrel{\circ}{\sigma}=\stackrel{\circ}{\bar{\Sigma}}+\alpha \mu(\stackrel{\circ}{\overline{\mathrm{E}}}-\stackrel{\circ}{\mathrm{E}} \mathrm{P})
$$

$\alpha$ is the scalar plastic accommodation function depending of $\nu$ and $\mu *$, where $\mu *$ is the instantaneous plastic shear modulus.

a decreases rapidly with increasing plastic flow from 1 to $10^{-2}$ or $10^{-3}$. Such estimation lowers the internal stress field.

The self consistent scheme can integrate the initial texture of the polycrystal; if $F(g)$ is the texture function, one has :

$$
\begin{aligned}
& \stackrel{\circ}{\mathrm{E}}=\langle\stackrel{\circ}{\bar{\varepsilon}}\rangle=F(g) \stackrel{\circ}{\frac{\rho}{\varepsilon}}(g) d g \\
& \frac{\circ}{\sigma}\left(g^{\prime}\right)=F(g) \frac{\circ}{\Sigma}+\alpha \mu\left(\frac{\circ}{\varepsilon} P(g)-\frac{\circ}{\varepsilon} P\left(g^{\prime}\right)\right) d g
\end{aligned}
$$

$g$ points the grains with a same orientation and $\bar{\sigma}\left(g^{\prime}\right)$ corresponds to the stress rate of the family of grains $g^{\prime}$ with all the other orientations.

These laws were extended to more complex situations such as anisotropic elasticity /7/. Nevertheless, no model has yet successfully taken into account some important parameters such as the grain size, the grain shape and the mechanisms of the plastic deformation induced by the grain boundaries (pile ups, inhomogeneities of the plastic deformation). In all cases these models led to an homogeneous plastic deformation of the grains, in contrast with reported experiments.

Loading tests carried out on polycrystals focused on the importance of the grain size in the macroscopic behaviour and the formation of inhomogeneous slip patterns inside the grain (the grains were plastified according to different slip patterns). Such inhomogeneities were observed in most metals and for grain sizes from about ten microns to some centimeters.

Many qualitative models devoted to describe the effect of grain size on stress-strain curves were published $/ 8 / / 9 / / 10 / / 11 / / 12 /$.

In most cases, these models considered the polycrystal as two phase materials (one phase constitued by the grain boundary layer harder than the other phase constituted by the grain bulk). Compared to the actual slip patterns, such representa- tions did not fit to the experiments. As a matter of fact, the size of the grain boundary layer must not be considered as a constant value inside the grain and the corresponding hardening law is not easy to determine experimentally.

\section{Macroscopic level analysis}

Uniaxial loading tests, carried out on various polycrystals showed two important features : firstly the inhomogeneity of the plastic glide inside the grains and secondly, the effect of grain size on the flow stress.

To give some illustration of the first effect, we have reported in figure (1) sketches of slip patterns observed in metals presenting different latent hardening properties and grain sizes /11/ /13/.
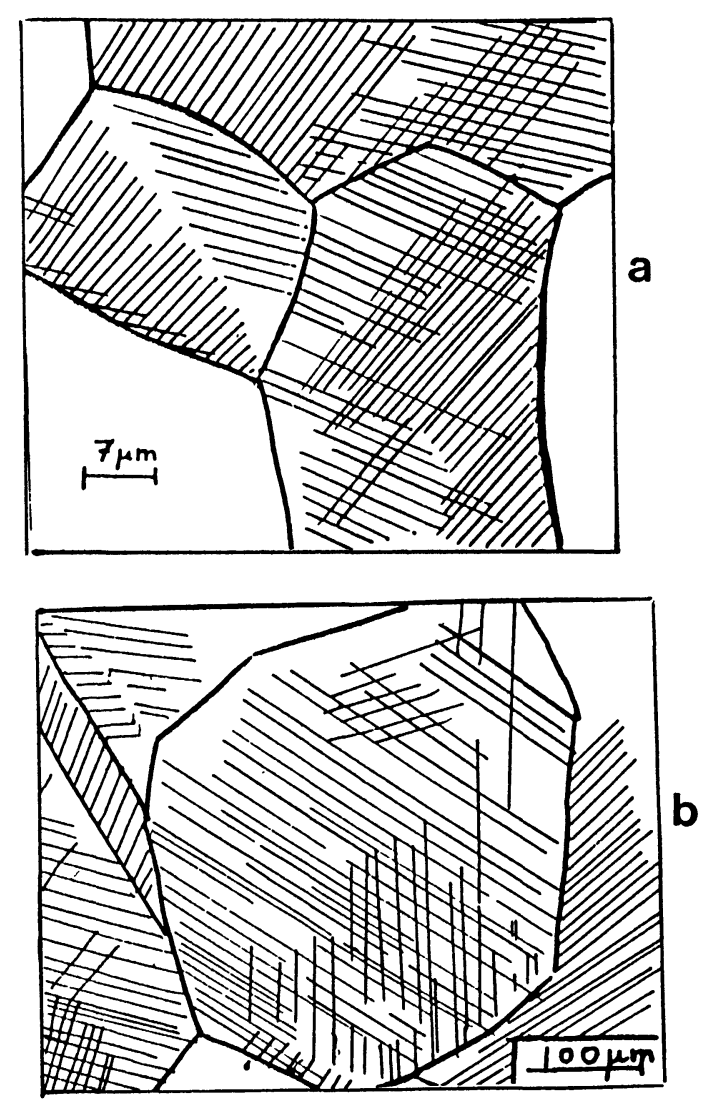

Fig. 1 : sketch of the slip pattern of

(a) an aluminiun polycristal strained up tol0\% (JAOUL $/ 11 /$ ).

(b) a 70-30 brass polycrystal strained up to $2.5 \%$ (DONG et al $/ 12 /$ ).

In most cases, various areas plastified by different slip systems, could be observed inside 
the same grain. Moroever, it could be noticed that only two slip planes could be activated in the same area of a grain. As a slip system initiated at the grain boundary could propagate inside the grains with increasing strain, it was not possible to separate unambiguously the behaviour of the grain interior from the behaviour of the grain boundary area.

Now, if we pay attention to experimental results about flow stress $\sigma$ expressed as a function of grain size $d$, we can use the Hall Petch law /15/ $/ 16 /$ for the yield stress:

$$
\sigma=\sigma_{0}+k d^{1 / 2}
$$

where $\sigma_{0}$ is the yield stress of the corresponding single crystal ; $k$ is a constant.

However we must emphasize that the law may be enlarged with care to flow stress. This law does not always fit to the experimental behaviour of all fcc metals $/ 10 /$. Generally the observed deviations from the law correspond to large grain sizes and large strain.

Some previous attempts to obtain a formulation of the flow stress as a function of grain size are based on two phase models :

Phase 1 corresponds to the grain interior whereas phase 2 concerns the boundary area.

If $\sigma_{1}$ and $\sigma_{2}$ are respectively the flow stress of the two phases, the macroscopic flow stress of the grain is determined by averaging the forces acting on the cross sections of the grain :

$$
\sigma S=\sigma_{1} S_{1}+\sigma_{2} S_{2}
$$

where $S_{1}$ and $S_{2}$ are the cross sections of the two phases respectively.

The grain interior contains what are usually called the statistical dislocations, whereas the grain boundary area contains pile ups of statistical dislocations as well as geometrical dislocations, the latter ones being activated in order to fulfill the compatibility conditions /8/ /15/. In this way, the grain boundary layer is harder than the bulk.

The main difficulty is to give a physical meaning to the four parameters $\sigma_{1}, \sigma_{2}, s_{1}, s_{2}$, which take into account the grain size d.To solve this difficulty, some models were proposed $/ 9 /, / 10 /$, $/ 11 /$. In Mecking's model, the grain interior flowstress is given by :

$$
\sigma_{1}-\sigma_{0}=M^{2} \theta \varepsilon
$$

$1 / M$ is the Schmid's factor of the grain

$\theta$ is a constant equal to $\mu / 200$ $\varepsilon$ is the applied strain.

The $M$ dependance of $\sigma$ means that the flow stress is a function of the relative orientation of the grain versus the tensile axis. Moreover the grain interior is assumed to be in a double slip situation.

The flowstress $\sigma_{2}$ of the grain boundary area is given as a function of the thickness layer $t$ by

$$
\sigma-\sigma_{0}=\alpha \mu\left(\frac{\varepsilon b}{t}\right)^{1 / 2}
$$

where $\alpha$ is a geometrical factor and b the Burger's vector amplitude.

The final formula is given by

$$
\sigma-\sigma_{0}=M^{2} \theta \varepsilon+\frac{4 t}{d} \quad \alpha \mu\left(\frac{b \varepsilon}{t}\right)^{1 / 2}-M \theta^{2} \varepsilon
$$

The difficulty is now to express the thickness $t$ as a function of $\mathrm{d}$ and $\varepsilon$. Generally one uses $t$ proportionnal to $d^{1 / 2}$ but no experimental determination has been yet performed to our knowledge. The reasons may be due to the misfit between the actual plastic strain distribution and the model one.

Note that these qualitative models cannot take into account neither the misorientations between grains nor the interaction phenomena between these grains.

In order to analyze with more accuracy the different mechanisms of the plastic deformation of aggregates, experimental studies were carried out at a more microscopic level.

3. Local analysis of effect of grain boundaries on the plastic flow of grains.

Although, at the microscopic level, grain boundaries can be considered as a source of dislocations and as an obstacle to the plastic glide (leading to pile ups of dislocations), at a medium level, which is now our framework, grain boundaries do not act by themselves. They act as interfaces between adjoining grains with different lattice orientations and consequently different plastic glide distributions which generally correspond to an incompatible plastic strain field and associated internal stresses.

Firstly, we sum up Kröner's formalism /16/ based on the continuum theory of dislocations used to calculate the internal stresses bound to an incompatibility of plastic strain and the main related results of the computations about the plane interfaces and junctions of grains. Secondly, we review some experimental works reporting the observed mechanisms of the plastic deformation in simple situations such as bicrystals, tricrystals and multicrystals. 
3.1. Incompatibility of plastic deformation. Internal stresses.

\subsubsection{Bicrystals}

Let us compare the two following situations : In the first case, a bicrystal composed of two grains named $I$ and II, separated by a plane interface normal to $x_{2}$, submitted to a uniaxial tensile loading along the axis $x_{3}$.

In the second case, two single crystals respectively identical with the two hereabove grains and tensile tested under the same conditions.

Except for specific orientations, each single crystal exhibits a single glide stage according to the primary slip system. At the onset of the plastic deformation, each grain of the bicrystal generally exhibits two slip systems:the primary and a secondary one, both being inhomogeneously distributed; in the grain the secondary slip system is usually concentrated near the grain boundary. The width of the boundary layer can reach half the grain. These secondary slip systems are generally said to ensure the compatibility conditions. As a matter of fact, it is not possible to build the bicrystal from the two plastically deformed single crystals. The misfit may be suppressed by some relevant addition of an extra elastic and plastic strain field (the latter field corresponding to the secondary slip systems).

Let us consider first the case of an elastic accommodation and let us suppose the bicrystal to be infinite and elastically isotropic.

If the initial distortions in the grains $I$ and II (say $\bar{\beta}^{\mathrm{PI}}$ and $\bar{\beta}^{\mathrm{pII}}$ respectively) are assumed uniform, the associated plastic incompatibility is superficial and localized at the grain boundary.

Note that $\bar{\beta}^{\mathrm{pI}}$ and $\bar{\beta}^{\mathrm{pII}}$ do not correspond to the actual deformation of the grains but rather of the corresponding isolated single crystals defined hereabove.

The dislocation density tensor $\alpha$ is defined by :

$$
\bar{\alpha}=-\operatorname{curl} \bar{\beta}^{\mathrm{p}} \quad \alpha_{1 j}=-\varepsilon_{i k 1} \beta_{1 j, k}^{p}
$$

and the incompatibility tensor $\eta$ is given by :

$\vec{n}=-$ Inc $\bar{\varepsilon}^{p} \quad \eta_{i j}=-\varepsilon_{i j k} \varepsilon_{j m n} \varepsilon_{1 m, k n}^{p}$ $\alpha$ and $\beta$ being related by :

$\eta_{i j}=\frac{1}{2}\left(\varepsilon_{i m n} \alpha_{j m, n}+\varepsilon_{j m n} \alpha_{i m, n}\right)$

$\varepsilon_{i j k}$ is the permutation tensor

${ }_{\beta} \mathrm{P}$ is the plastic distortion at any point of the bicrystal. In the case of the two differently plastified grains
$\overline{\mathrm{B}}^{\mathrm{P}}=\overrightarrow{\mathrm{B}}^{\mathrm{PI}}+\overline{\Delta \bar{\beta}}^{\mathrm{P}} \mathrm{Y}\left(\mathrm{x}_{2}\right)$

with $\Delta \vec{\beta}^{\mathrm{P}}=\overline{\bar{\beta}}^{\mathrm{pII}}-\overline{\bar{\beta}}^{\mathrm{pI}}$

$\mathrm{Y}\left(\mathrm{x}_{2}\right)$ is the Heaviside function

$\vec{x}_{2}$ is the normal to the plane grain boundary.

Let $\bar{\beta}$ be the extra elastic field distortion. The total plastic distortion $\bar{\beta}^{T}=\bar{\beta}^{p}+\bar{\beta}^{e}$ is compatible if

$\operatorname{curl} \bar{\beta}^{\mathrm{T}}=0$ or $\quad \operatorname{curl} \vec{\beta}^{\mathrm{e}}=\alpha$

The compatibility conditions can be expressed as a function of the strain fields $\bar{\varepsilon}^{\mathbf{e}}$ and $\bar{\varepsilon}^{\mathrm{p}}$ only by

Inc $\bar{\varepsilon}^{\mathbf{e}}=-$ Inc $\bar{\varepsilon}^{\mathrm{p}}=\bar{\eta}$

The internal stresses are deduced from $\vec{\varepsilon}$ by the Hooke's law.

In the case of an infinite bicrystal, described by $(3.4)$, the internal stress field is calculated as :

$\sigma_{11}=-\frac{\mu}{1-\nu}\left(\Delta \varepsilon_{11}^{\mathrm{p}}+\nu \Delta \varepsilon_{33}^{\mathrm{p}}\right) \operatorname{sgnx}_{2}$

$\sigma_{33}=-\frac{\mu}{1-v}\left(\Delta \varepsilon_{33}^{\mathrm{p}}+\nu \Delta \varepsilon_{11}^{\mathrm{p}}\right) \operatorname{sgnx}_{2}$

$\sigma_{13}=-\mu \Delta \varepsilon_{13}^{\mathrm{p}} \operatorname{sgnx}_{2}$

$\sigma_{12}=\sigma_{22}=\sigma_{23}=0$

sgnx ${ }_{2}$ denotes the sign of $x_{2}$.

This stress field is uniform in each grain and presents a discontinuity at the grain boundary.

3.1.2. Junction of three grains

The same formalism can be applied to a crystal constituted by three grains (named I, II, III, respectively) separated by three plane grain boundaries with a common junction parallel to the oz axis $/ 18 /$.

Let us consider the theoretical situation of three grains with a uniform plastic strain characterized by $\bar{\varepsilon}^{\mathrm{pI}}, \bar{\varepsilon}^{\mathrm{pII}}$ and $\bar{\varepsilon}^{\mathrm{pIII}}$ respectively.

The strain field at any point $p$ defined by $r$ and $\Theta$ parameters (figure 3 ) can be written as follows : $\bar{\varepsilon}^{\mathrm{P}}(\mathrm{r}, \theta)=\bar{\varepsilon}^{\mathrm{pI}}+\sum_{1} \Delta \bar{\varepsilon}^{\mathrm{pI}} \mathrm{Y}\left(\theta-\theta_{1}\right) \mathrm{Y}(\mathrm{r})$

where $\Delta \bar{\varepsilon}^{\mathrm{p}}$ is the strain discontinuity at the interface (i)

$\theta_{i}$ is the position of the (i) interface versus the $0 x y z$ axes system

$Y\left(\theta-\Theta_{1}\right)$ and $Y(r)$ are Heaviside's functions.

The deduced internal stress field is given by an analytical expression :

$\bar{\sigma}(\mathrm{r}, \theta)=\overline{\mathrm{F}}\left(\mu, \nu, \Delta \bar{\varepsilon}^{\mathrm{p}}, \theta^{i}, \theta\right)+\bar{G}\left(\mu, \nu, \Delta \varepsilon^{\mathrm{p}}, \theta^{i}\right) \log \mathbf{r}$

$\overline{\mathrm{F}}$ and $\overline{\mathrm{G}}$ are regular functions.

The second term corresponds to large internal stresses near the triple point. But it is not possible to determine their range, the calculation 
being carried out assuming an infinite medium. Far from the triple node it is physically justified to consider only the first term.

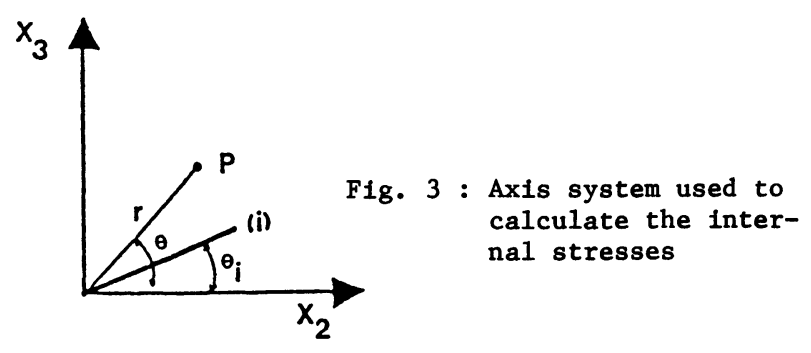

These two solutions must be used with care:

- They assume a perfect match between the grains and exclude any grain boundary sliding.

- They describe an internal stress field in infinite media, ignoring edge effects in finite bicrystals or tricrystals.

- Moreover they assume a uniform strain field $\bar{\varepsilon}^{p}$ inside the grains.

The plastic flow inside the grains, the evolution of $\bar{\varepsilon}^{\mathrm{p}}$ by activation of new slip systems as well as the desactivation of the former ones may be taken into account through a step by step calculation.

In the case of flowing bicrystals or tricrystals a good approximation consists either in replacing the elastic shear modulus $\mu$ in formulae (3.7) and (3.9) by the overall elastic- plastic secant modulus $\mu^{8}$ for the case of monotonic radial loading or in replacing both $\bar{\sigma}$ and $\bar{\varepsilon}^{\mathrm{P}}$ by the rates $\dot{\sigma}$ and $\frac{2}{\varepsilon}$ and $\mu$ by the tangent elastic-plastic modulus $\mu * / 5 /$ 171 .

The nature of the slip systems successively activated in the grains is determined as follows :

Let us assume that $k^{I}$ and $k^{I I}$ are the two first slip systems activated in grains $I$ and II respectively by the applied stress $\Sigma$ for the given macroscopic strain E. One has :

$\varepsilon_{1 j}^{p}=\frac{1}{2}\left(m_{i j} n_{j}+m_{j} n_{i}\right) \gamma$

where $\vec{m}$ and $n$ are unit vectors parallel to the slip direction and normal to the slip planes.

$\gamma$ is the crystallographic shear strain and $\gamma$ depends upon the macroscopic strain $E$.

For a given increment of the macroscopic strain $d E$ the slip system $g^{I}$ will be activated in grain $I$ if

$$
\mathrm{d} \tau^{\mathrm{g}}=\overline{\mathrm{R}}^{\mathrm{g}}(\mathrm{d} \bar{\sigma}+\mathrm{d} \overline{\mathrm{L}})>\sum_{\mathrm{k}} \mathrm{H}^{\mathrm{kg}} \mathrm{d} \gamma^{\mathrm{k}}
$$

$\tau^{g}$ is the resolved shear stress on the $g$ slip syssystem

$\mathrm{R}^{\mathrm{g}}=\overrightarrow{\mathrm{m}} \mathrm{g} \cdot \overrightarrow{\mathrm{n}} \mathrm{g}$ $\bar{\sigma}$ is the internal stress field due to $k^{I}$ and $k^{I I}$ plastic incompatibility

$\bar{\Sigma} \quad$ is the applied stress field

$\overline{\mathrm{H}} \quad$ is the hardening matrix.

The $\mathrm{H}^{\mathrm{kg}}$ components correlate the slip activity of the $g$ slip system with the hardening behaviour of the already active $\mathrm{k}$ slip systems.

If the $g$ slip system fulfils the condition (3.11) it must be taken into account in the next step of the calculation.

3.2. Elastic anisotropy and internal stresses.

In the microplasticity stage initiation of slip systems at the grain boundaries and their further localization in a narrow area adjoining the grain boundarles were noticed by different authors /19/ /20/. Elastic anisotropy and correlated internal stresses were often assumed to be at the origin of the activation of these slip systems.

Evaluations of these internal stresses were derived from Finite Elements Method in bicrystals /21/, tricrystals /22/and multicrystals /23/ for various anisotropy factors and size factors. The main results were :

- In bicrystal case, the internals stresses, were never higher than twice the applied stress near the grain boundary.

- In multicrystal case,an increase of internal stresses could be noticed close to quadruple or triple nodes. At low strain,the activation of extra slip systems due to an anisotropy effect in the vicinity of the grain boundaries can contribute to harden them and accordingly justify some two phases models.

A lack of a similar computation in plasticity does not allow to compare the relative importance of anisotropy and plastic incompatibility effects. Nevertheless anisotropy effects are not likely to rule the slip patterns observed at medium and large strains.

3.3. Effect of a plane interface on the formation of plastic inhomogeneities.

\subsubsection{Bicrystals}

Observations carried out on copper and aluminium bicrystals /24/ $/ 25 /$ with a plane grain boundary, tensile tested at low temperature showed the onset of a domain, near the grain boundary, exhibiting a plastic strain different from the whole grain. Moreover, different slip patterns could be seen on the two faces perpendicular to the grain boundary. 
Note that the activation of some small slip lines close to the grain boundary in the microplasticity stage were sometimes observed in copper bicrystals. These phenomena could be related to an anisotropy effect but in all cases they were screened by other ones due to plasticity.

During the plasticity stage the activation of slip systems with increasing strain matched the following scheme :

- activation of the primary slip systems (higher Schmid factor) far from the grain boundary and then propagation towards it. The primary slip system reached the grain boundary on one of the sample face and stopped at some distance of 1 it on the other face giving rise to a domain. This domain was usually limited by the grain boundary and what we called the front of plastification. The width of the domain on the observation fact was varying with the relative orientation of the grain boundary and the slip direction.

- growth of a secondary slip system in the domain.

As the strain was increased, two different situations were observed :

- the intragranular interface between the two slip systems was stable. Other slip systems were activated on both sides of the intragranular interface as well as the grain boundary, in order to relax all internal stresses. The slip pattern stayed inhomogeneous up to rupture and large lattice misorientations could be measured in a same grain.

- the intragranular interface vanished due to some propagation of the primary and the secondary slip systems in each other. The slip pattern then turned homogeneous (double slip).

Formation of such domains was not a superficial effect : observations of the cross section of a plastically deformed bicrystals after a chemical etching showed that the domain concerned all the grain boundary area.

The formation of domains in bicrystals could not be explained from any incompatibility of strain (domains could be observed in compatible bicrystals). On the contrary the nature and the evolution of the intragranular could be predicted by the internal stress calculation reported in section (3.1.1).

The formation and the geometrical localization of domains was only function of the relative orientation of the primary slip direction with res pect to the grain boundary. The size of the domain was also a function of the width of the bicrystal.

A rough model was proposed to describe the formation of the intragranular interfaces /24/ /25/ :

At the very beginning of the plastic strain, the primary slip system is not homogeneously activated. The corresponding slip lines are more dense far from the grain boundary in areas where the average free path of dislocations is high.

The grains are progressively plastically deformed according to the propagation of slip bands in the slip directions, the distance between the bands decreasing with increasing strain. Such propagation cannot induce internal stresses. Consequently, the front of plastification is a plane normal to the slip direction or normal to the slip plane.

When the fronts of plastification impinges the grain boundary, the propagation turnes incompatible and its rate decreases. New slip systems are then activated between the grain boundary and the front of plastification. The initial position of the Intragranular interface can be deduced from crystallographical considerations.

This process is illustrated on figures (4) and (5).

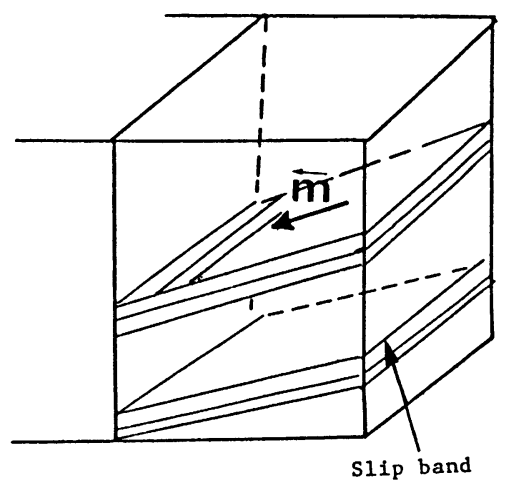

Fig. 4 : Bicrystal without domain.

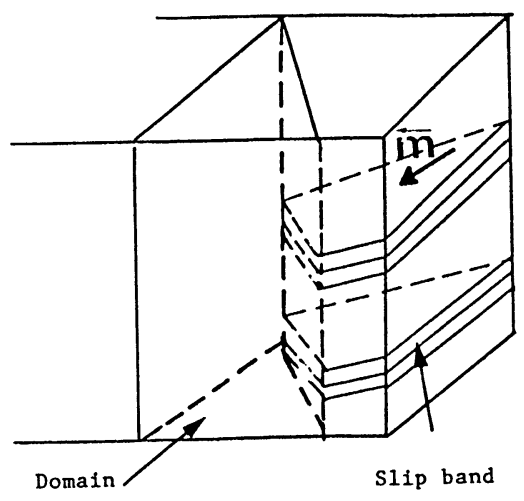

Fig. 5 : Bicrystal presenting a domain. 
On these figures two extreme cases are reported. In the first case, the angle $\theta$ between the slip plane and the grain boundary is large. The front of plastification propagates up to the grain boundary. There is no formation of domain. In the second case $\theta$ is weaker than 60 degrees. The front of plastification is pinned by the grain boundary and a domain is built.

The nature of slip systems activated in the domaln is determined from an internal stress calculation similar to section (3.11) but taking into account the intragranular interfaces and the grain boundary in a similar way. In some bicrystals the effect of intragranular interfaces was higher than the grain boundary one. In all analyzed bricrystals, the observed secondary slip system the had the highest resolved shear stress.

At a further stage of the straining we had to take into account the latent hardening properties of the relevant metal. The slip systems $g$ activated in the domain or in the remaining grain generally had the higher $\tau^{\mathrm{g} / \mathrm{H}^{\mathrm{kg}}}$ ratio. This means a high resolved shear stress and a low interaction with the previously activated slip systems in the concerned area. Note that no more than two slip systems were observed in a given area of a grain.

3.2.2. Junctions of grains.

Copper and zinc tricristals presenting three intersecting plane grain boundaries were tensile tested at room temperature.

Observations carried out on the two faces perpendicular to the junction showed two kinds of intragranular interfaces named $A$ and $B$ respectively :

- interface (A) limited large domains similar to those observed in bicrystals.

- interface (B) appeared at the triple node as an extension of the opposite grain boundary and vanished inside the grain. Slip systems were different on both sides of the interface and induced lattice misorientations inside the grain (fig. 6). With increasing strain, the density of slip lines increased near the node faster than inside the grain.

The conditions of formation of intragranular interfaces (B) are not very clear. As a matter of fact, these interfaces rise at the very beginning of the deformation, as the activation of slip systems is still weak. Explanations based only on plastic incompatibility do not hold.

On the contrary, evolution and stability of $B$ interfaces can be analyzed from plastic compatibility conditions. According to the calculation presented in section 3.1., assuming an homogeneous plastic strain of the grains, the internal stresses present a very large value near the node. This means that the starting assumption (homogeneous plastic strain) is not physically correct. One may lower the internal stresses and to cancel the coefficient of the logarithmic term in expression (3.9) by adding some new (B) interfaces and extra slip systems.
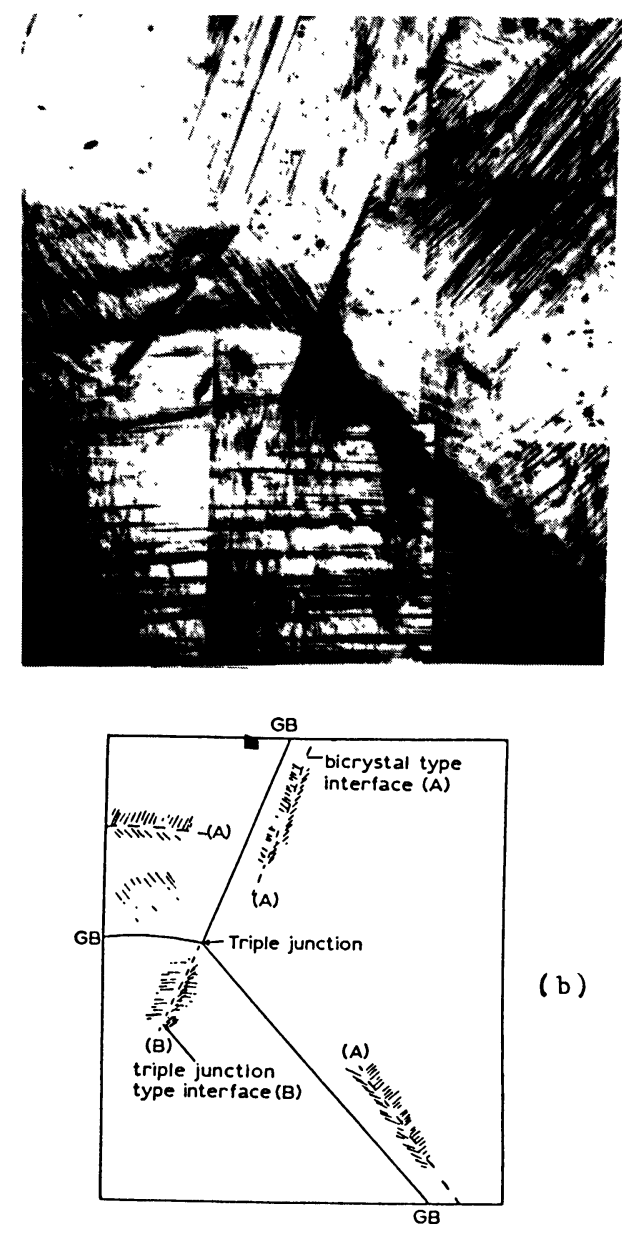

(b)

Fig. 6 : (a) Copper tricrystal presenting two kinds of intragranular interfaces (A) and $(B)$.

(b) Sketch of the tricrystal.

These experimental results leads to assume that the inhomogeneities observed in tricrystals are correlated with the strong internal stress field bound to the node.

At a more microscopic level, the mechanism of plastic deformation of a grain was observed in situ by TEM $/ 26 /$. The sequences were: firstly activation of lattice dislocations, secondly activation of triple point sources and formation of pile-ups, 
thirdly propagation of slip across some part of the grain boundaries by dislocation generation induced by stress concentration at the pile ups.

It was not easy to compare these observations with our work, carried out on different levels; nevertheless they agreed on two points : the plastic deformation appeared inside the grain (not at the grain boundaries) and the triple nodes had an important effect on the plastic deformaticn.

\subsubsection{Multicrystals}

In order to have a better understanding of the nature of actual interactions (long range or short range internal stress-field) between grains, zinc and copper multicrystals presenting about ten grains with grain boundaries normal to the large faces of the samples were tensile tested at room temperature.

The plastic inhomogeneities inside the grains were characterized by their superficial strain field and their lattice rotation field. These two fields were measured with fiducial grids and thanks to Kossel's technique $/ 18 /$.

We have reported in figure 7 , a map of the local strain fleld component Ezz ( $\mathrm{Oz}$ being parallel to the tensile axis), in order to illustrate the formation of plastic inhomogeneities.

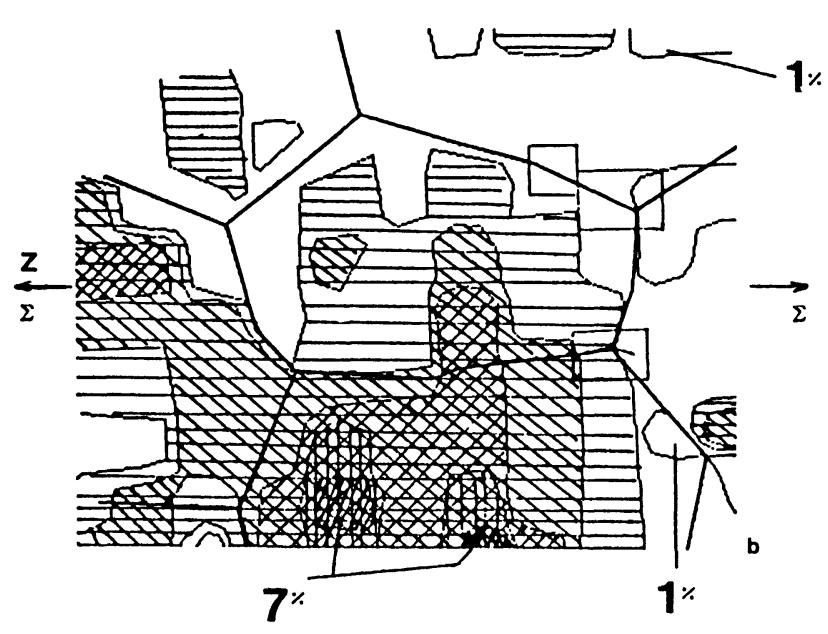

Fig. 7 : Map of local deformation in a copper multicrystal presenting plastic inhomogenities.

The results can be summarized as follows :

- Plastic deformation did not initiate systematically at the grain boundaries. Large areas on each side of some grain boundaries were plastically deformed less than $1 \%$, the macroscopic strain $\mathrm{E}$ being up to $3 \%$. These large undeformed domains had a crystallographic origin and were similar to bicrystals' ones.

- Junctions had a small range effect compared to the grain size.

- In most of the observation cases, the strain was continuous on each side of the grain boundaries, but presented variation of magnitude along them. This effect, never observed in bicrystals might be due to junctions.

Our purpose is now to determine the actual range of the internal stress bound to grain boundaries and junctions, by analyzing the nature of slip systems activated in multicrystals of different grain size.

\section{Conclusion}

This paper is an attempt to classify some of the physical approaches concerning the behaviour of polycrystals under loading.

An other way of modelling the polycrystal plasticity has been presented : by a step by step investigation starting from the single crystal case and then successively examining more complex situations such as the bicrystal as well as tricrystal cases, we have succeeded in analyzing the initiation and the growth of plastic inhomogeneities inside the grains. Though our procedure has not yet allowed us to completely describe the plastic behaviour of polycrystals, we hope to succeed by introducing more parameters in our step by step approach.

\section{References}

/1/ Hirth, J.P., Metall. Trans. $\underline{3}$ (1972) 3047-3067.

/2/ Eshelby J.D., Proc. Roy. (London), A 241,376, (1957)

/3/ Eshelby J.D., Progress in Solids Mechanics (1961) Vol II, J,N Sneddon and R. Hill (Eds), North-Holland, Amsterdam, p. 87

/4/ Kröner E., Zur plastischen Verformung des Vielkristalls, Acta Metall 9, 155-162 (1961)

/5/ Hill R., J. Mech. Phys.Sol. 13, 89,101 (1965)

/6/ Bervelller M., Zaoul A., J. Mech. Phys Solids. 26, 325 (1979)

/7/ Bervelller M., Zaoul A., Journ. de Mécanique $19,2,343-361,(1980)$

/8/ Ashby M.F., Phil. Mag. 21, 399 (1970)

/9/ Thomson A.W., Baskes M.I., Flanagan W.F., Acta Metall. 21, 1017-1028 (1973)

$/ 10 /$ Mecking H, , Proc. 5th Int. Cong. Strength of Metals. and Alloys. (Edited by P. Haasen et a1) p. 1573 Pergamon, Oxford (1979) 
/11/ Meyers M.A., Phil. Mag. A 46, 5, 737-759 (1982)

/12/ Armstrong R.W., Codd I., Douthwatte R.M. and Petch N.J., Phil. Mag. 11, 1135 (1962)

/13/ Dong H., Thompson A.W., Metall. Trans. 16A, 1025-1030 (1985)

/14/ Jaoul B., Etude de la plasticité et application aux matérlaux, Dunod, Paris (1965)

/15/ Ha11 E.0., Proc. Phys. Soc. (London) 64B 747, (1951)

/16/ Petch N.J., J. Iron Steel Inst. 174, 25 (1953)

/17/ Kröner E., Kontinuums Theorie der Versetzungen und Eingenspannungen, Berlin, Springer Verlag (1958)

/18/ Rey C., Mussot P., Zaoul A., Grain Boundary structure and Related Phenomena, Proceedings of JIMIS 4, (1986) Supplement to transactions of the Japan Institute of Metals p. 867

/19/ Hashimoto K., Margolin H., Acta Metall. 3l-5, 773-785 (1983) Acta Metali. 31-5, 787-800
(1983)

/20/ Hook R.E., Hirth J.P., Acta Metall. 15, 535, 1099 (1967)

/21/ Kitagawa K., Asada H., Monzen R., Kikuchi M., grain Boundary Structure and Related Phenomena, Proceedings of JIMIS-4 (1986) supplement to transactions of Japan Institute of Metals

/22/ Kurzydlowski K., Celinki Z., Grabski M.W., Res. Mechanica 1 283-287 (1980)

/23/ Ohashi T., grain Boundary Structure and Related Phenomena, Proceedings of JIMIS 4 (1986). Supplement to Transaction of Japan, Institute of Metals.

/24/ Rey C., Zaoui A., Acta Meta11. 30, 523-535 (1982)

/25/ Rey C., Zaou1 A., Acta Metall. Lo, bo/-oy/ (1980)

/26/ Kurzysdlowski K.J., Varin R.A., Zielinski W., Acta Metal1. 32, 71-78, (1984) 